Revista Brasileira de

Engenharia Agrícola e Ambiental

v.16, n.12, p.1374-1380, 2012

Campina Grande, PB, UAEA/UFCG - http://www.agriambi.com.br

agriambi Protocolo 274.11 - 24/11/2011 • Aprovado em 24/08/2012

\title{
Desempenho de rede de sensores sem fio em casa de vegetação
}

\author{
Barbara Teruel $^{1}$, Elaine C. de S. Alves ${ }^{1}$, Clerivaldo J. Roccia ${ }^{2}$, \\ Francisco J. Arnold ${ }^{3}$ \& Leonardo L. Bravo-Roger ${ }^{3}$
}

\begin{abstract}
RESU MO
A potência de sinal é um importante parâmetro a ser considerado em um projeto de implantação de rede de sensores sem fio no meio agrícola. Este trabalho tem como objetivo coletar dados de qualidade de sinal transmitido entre uma estação base e um nó sensor situado no interior de uma casa de vegetação, localizada no campo experimental da Faculdade de Engenharia Agrícola - UNICAMP, com o auxílio de ferramentas computacionais. Após a implantação do nó sensor foram realizados experimentos com vista a se verificar alcance e qualidade do sinal de acordo com sua potência. Através desses ensaios foi possível concluir que uma potência de sinal com valor de $-12,2 \mathrm{dBm}$ é suficiente para manter a transmissão entre a estação base e nó sensor. Testes também foram realizados com uma potência de sinal de $-9,2$ $\mathrm{dBm}$ e mostraram que a rede possuía qualidade classificada "muito alta" porém, através do teste de duração da bateria, nota-se que existe economia de energia ao se utilizar a potência de sinal de $-12,2$ $\mathrm{dBm}$. Foi avaliada a influência que o valor da potência de sinal tem sobre a qualidade do sinal de transmissão e recepção, o gasto de bateria e o alcance do sinal.
\end{abstract}

Palavras-chave: qualidade de sinal, atenuação, agricultura, Fragaria ananassa Duch

\section{Performance of wireless sensor network in a greenhouse}

\begin{abstract}
A B ST RAC T
The signal strength is an important parameter to be considered in a project to establish network of wireless sensors in an agricultural environment. This work aims to collect quality data signals transmitted between a base station and a sensor node located inside a greenhouse, which is an experimental field located in the College of Agricultural Engineering - UNICAM P, with the help of computational tools. After sensor node deployment, experiments were performed in order to check range and signal quality. Through these experiments, it was concluded that a radiofrequency power value of $-12.2 \mathrm{dBm}$ is sufficient to maintain transmissions between base station and the sensor. Tests were also performed to a radiofrequency power of $-9.2 \mathrm{dBm}$, and showed that the network had quality rated as "very high". However, by testing the battery life, it is noted that there is an energy saving when the radiofrequency power of $-12.2 \mathrm{dBm}$ is used. It was shown that the value of the signal strength has an influence on the quality of signal transmission and reception, battery depletion and signal range.
\end{abstract}

Key words: signal quality, attenuation, agriculture, Fragaria ananassa Duch

\footnotetext{
FEAGRI/U NICAMP. Av. Cândido Rondon, 501, Barão Geraldo, CEP 13083-875, Campinas, SP. Fone: (19) 3521-1082. E-mail: barbarat@feagri.unicamp.br; elaine.cangussu@gmail.com;

${ }^{2}$ Escola de Engenharia de Piracicaba. Av. M onsenhor M artinho Salgot, 560, Bairro Areão, CEP 13414-040, Piracicaba, SP. E-mail: cjroccia@hotmail.com 3 Faculdade de Tecnologia/U NICAMP, R. Paschoal Marmo, 1888, Jd. Nova Itália, CEP 13484-332, Limeira, SP, Fone: (19) 2113-3492. E-mail: arnold@ft.unicamp.br; leobravo@ft.unicamp.br
} 


\section{INTRODUÇÃO}

$\mathrm{Na}$ medida em que na agricultura ocorrem avanços tecnológicos, intensifica-se o uso de diferentes aparelhos eletroeletrônicos e de modelos de negócio, a fim de melhorar a qualidade dos produtos, aumentar a produção, reduzir custos e diminuir o impacto ao meio ambiente. Neste contexto, a medição das variáveis envolvidas nas diferentes cadeias agrícolas torna-se indispensável para gerar as informações necessárias na tomada de decisão, tanto em sistemas de controle (Queiroz et al., 2008) dos processos envolvidos como em sua gestão e monitoramento.

Além disso, diversos processos agrícolas precisam de mobilidade das redes de sensores devido à existência de limitações para o uso de cabos e fios e a necessidade de monitorar regiões extensas e afastadas. Esta é uma área onde Redes de Sensores Sem Fio (RSSF) ganham espaço, inclusive em aplicações subaquáticas (Santos et al., 2010) por suas características intrínsecas de mobilidade, transmissão sem fio e facilidade de instalação.

Segundo Matijevics \& Simon (2010) avanços contínuos na tecnologia sem fio possibilitaram a implantação de redes de sensores para monitorar vários aspectos do ambiente agrícola. A utilização de RSSF na agricultura oferece novos recursos, tanto para controle quanto para monitoramento e transmissão de dados. Recentes avanços nesta área têm levado ao desenvolvimento de aparelhos com baixo custo, baixo consumo de energia e de novos nós sensores multifuncionais (RuizAltisent et al., 2010).

Especificamente no caso de casas de vegetação, o uso de RSSF é uma alternativa para obtenção de dados em tempo real, os quais são importantes para gerar informações que auxiliem no controle e no monitoramento do sistema garantindo um ambiente adequado para a cultura.

Morais et al. (2004) e Jackson et al. (2007) mostraram a viabilidade do uso de RSSF para monitoramento de umidade do solo. Outros autores já tratam do uso de RSSF para aquisição de dados utilizados no desenvolvimento de sistemas de controle de que são exemplos os trabalhos realizados por Serôdio et al. (2001) e Omid \& Shafaei (2004).

Entretanto, ainda são muitos os desafios para a adequação deste tipo de rede de sensores na área agrícola. Muitas casas de vegetação utilizam redes de sensoriamento cabeadas em que, em geral, fontes de energia elétrica para alimentar os dispositivos estão disponíveis. Todavia, essas redes apresentam limitações em termos de mobilidade dos sensores conectados aos cabos. Contrariamente, a mobilidade dos sensores representa uma das potencialidades do uso de RSSF, mas tem, como desvantagem, a limitação de autonomia energética para alimentação dos nós (Wang et al., 2006).

Desta forma, e mesmo em ambientes controlados, como em casas de vegetação, o problema do consumo de energia dos nós nas RSSF permanece. Alguns experimentos demonstram que o processo de transmissão de dados em uma RSSF gasta muita energia, enquanto o processamento de dados consome significativamente menos (Anastasi et al., 2009). Portanto, o gerenciamento de energia e a obtenção de valores ótimos da potência a ser utilizada no processo de transmissão, são indispensáveis.

Morais et al. (2008), por exemplo, com uma RSSF em vinhedo, usaram como fonte energética um conjunto de equipamentos para a captação de energia solar, do vento e, até mesmo, da água de irrigação. Outro obstáculo da implantação de sensores sem fio na agricultura é a atenuação de sinal causada pela presença de vegetação. A atenuação ocorre, principalmente, porque a frequência de transmissão da RSSF é de 2,4 GHz, frequência esta absorvida por materiais ricos em água, como plantas, por exemplo.

Neste trabalho os experimentos foram desenvolvidos junto a um cultivo de morangos (Fragaria ananassa Duch), assim como fizeram Aziz et al. (2009) que desenvolveram um sistema com RSSF para monitorar as variáveis ambientais de uma casa de vegetação para esta mesma cultura. Os resultados desses testes indicaram que o sistema desenvolvido foi confiável para a propagação dos dados em diferentes condições.

Esta monitoração pode trazer vantagens como: facilidade do controle de pragas e doenças, a possibilidade de produção em períodos de entressafra e o aumento da produtividade, porém para o controle do ambiente em casas de vegetação o uso de sensoriamento com cabos é ainda o mais comum entre os produtores brasileiros. Assim, torna-se atraente a opção de utilização de rede de sensores sem fio (RSSF) no lugar do cabeamento. Objetivou-se, portanto, neste trabalho, realizar um estudo sobre a utilização de RSSF em casas de vegetação, através da análise de dados de qualidade, gasto de energia e alcance do sinal.

\section{Material e MÉTOdos}

A casa de vegetação, em que o experimento foi realizado, está situada no Campo Experimental da Faculdade de Engenharia Agrícola - UNICAMP; trata-se de uma estrutura metálica de $6,5 \mathrm{~m}$ de largura por 11,0 m comprimento, 3,0 $\mathrm{m}$ de pé-direito e 2,0 $\mathrm{m}$ de altura acima do pé direito. O telhado é do tipo duas águas, com inclinação de $31,6^{\circ}$, sendo o material lateral e do telhado constituído por plástico transparente difusor de luz (PEBD - polietileno de baixa densidade) com $150 \mu \mathrm{m}$ de espessura, tratado contra raios ultravioleta; as laterais da casa de vegetação possuem muretas de concreto com altura de 0,30 m e largura de $0,10 \mathrm{~m}$.

No interior da casa de vegetação foram fixadas 4 bancadas que sustentam o sistema hidropônico para os morangos (Figura 1). Cada bancada possuía um total de seis canaletas feitas com tubos de PVC (meio dielétrico). Em cada canaleta foram enfileirados 16 vasos plásticos com morangos em fase de frutificação. Nas canaletas está presente um fluxo contínuo de água (meio condutivo). Assim, a água representa uma possível fonte de reflexão e atenuação de ondas eletromagnéticas.

Além das bancadas, o centro da casa de vegetação possuía uma bandeja de fibra de vidro com dimensões de 1,40 por 1,10 $\mathrm{m}$, colocada numa altura de $0,30 \mathrm{~m}$ do piso, na qual foram distribuídos 60 vasos com morangueiros que, por sua vez, tinham altura média de copa de $0,2 \mathrm{~m}$ e massa média de $450 \mathrm{~g}$. 


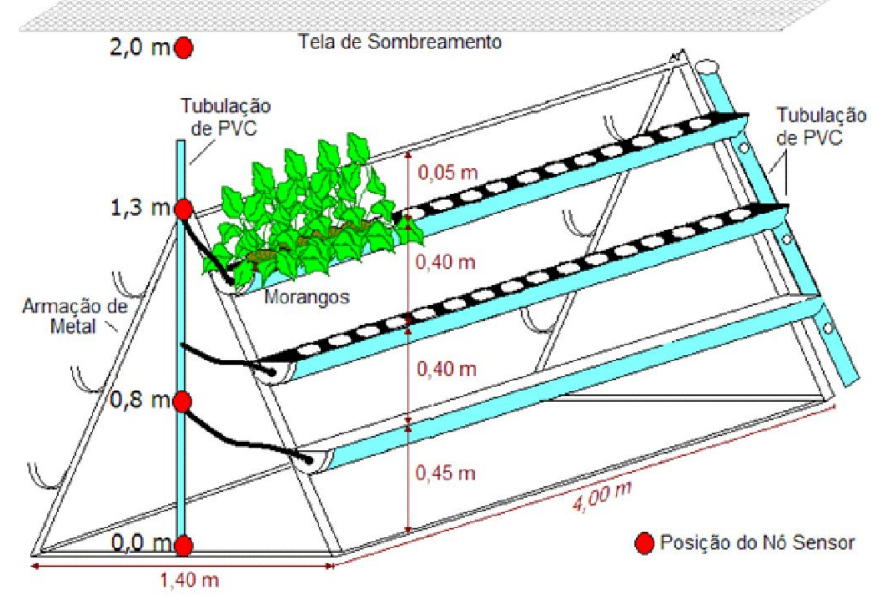

Figura 1. Bancada com indicação do posicionamento do nó sensor

Na Figura 2 é possível observar a localização da bandeja, das bancadas, do ventilador e da porta.

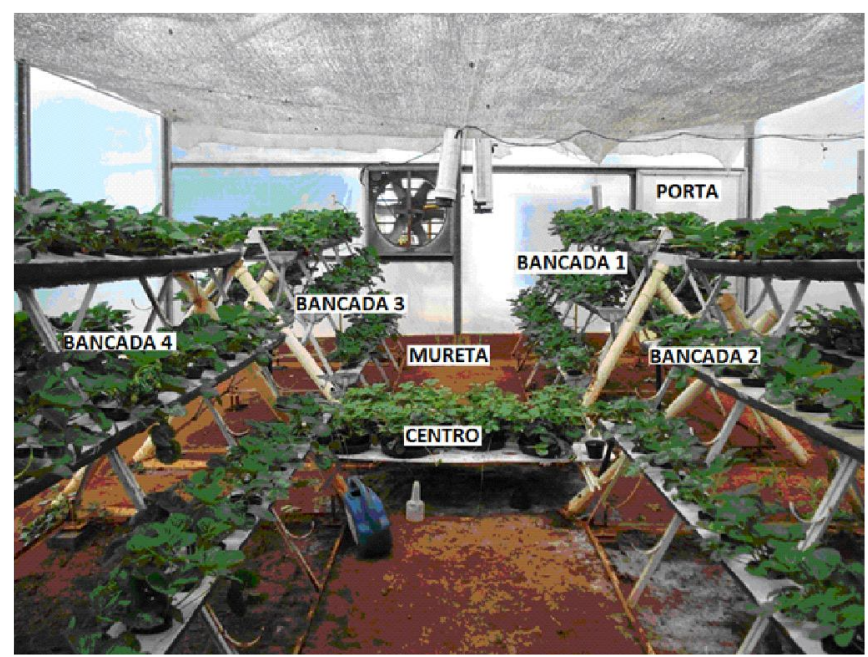

Figura 2. Vista interna da casa de vegetação com porta, bancadas, mureta da frente, bandeja do centro e ventilador

A RSSF utilizada é da marca Crossbow®, opera na frequência de 2,4 GHz e é formada por módulos de programação, placas, sensores para medição de temperatura, umidade relativa, sistema de aquisição e processamento de dados, e nós sensores. Em todos os testes a rede foi configurada no modo High Power (HP).

Foram utilizados nos experimentos a placa de aquisição MDA300CA, os gateways MIB600 e MIB 520, a placa OEM hospedeira e os softwares MoteView ${ }^{\circledR}$ além do MoteConfig® (Figura 3). A Estação Base, formada pelo computador, pelo MIB 600 e pelo MIB 520, foi instalada em uma sala próxima da casa de vegetação e fixada numa altura de $1,3 \mathrm{~m}$ em relação ao piso.

A placa de aquisição MDA300CA (Figura 4A) foi desenvolvida para as plataformas MICA2, MICAZ e IRIS e possui um sensor SHT15 da Sensirion $\AA$. Este sensor mede a umidade relativa através de um elemento capacitivo enquanto a temperatura é medida por semicondutor.

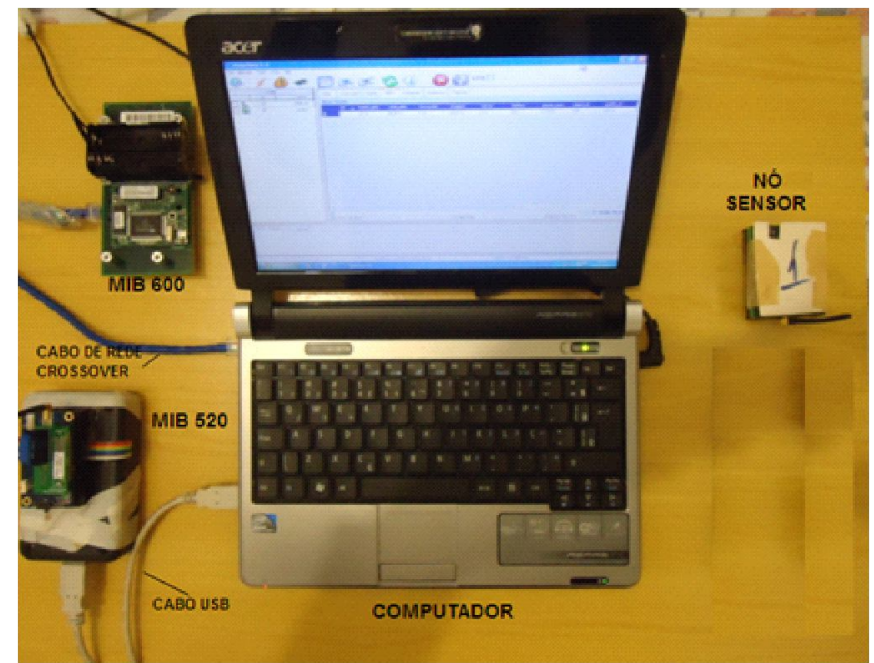

Figura 3. Componentes da estação base

A plataforma do nó sensor é a IRIS® (Figura 4B) opera na frequência de 2,4 GHz e foi projetada especificamente para incorporação em Redes de Sensores sem Fio. A taxa de envio de dados é de $250 \mathrm{kbps}$ e, como fonte de energia, foram utilizadas duas pilhas do tipo AA.

A.

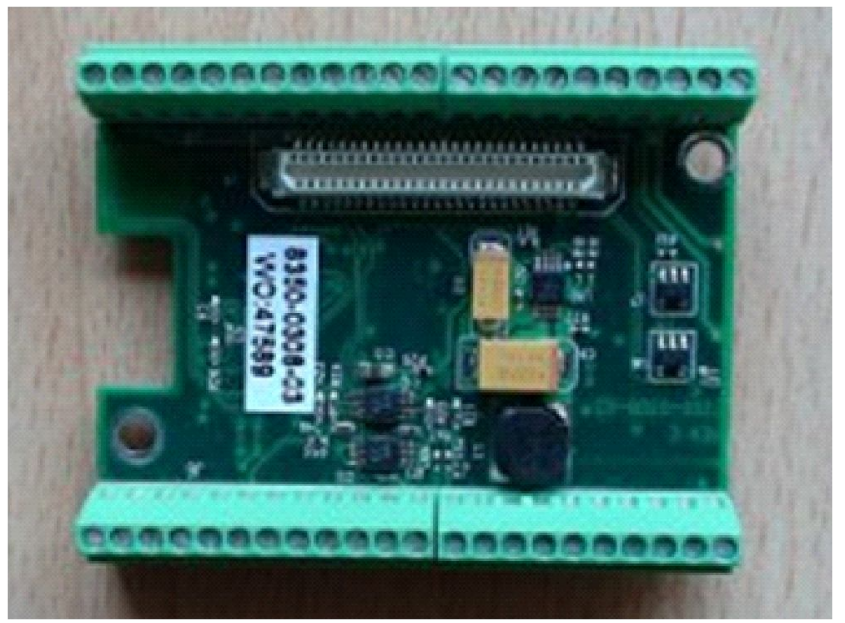

B.

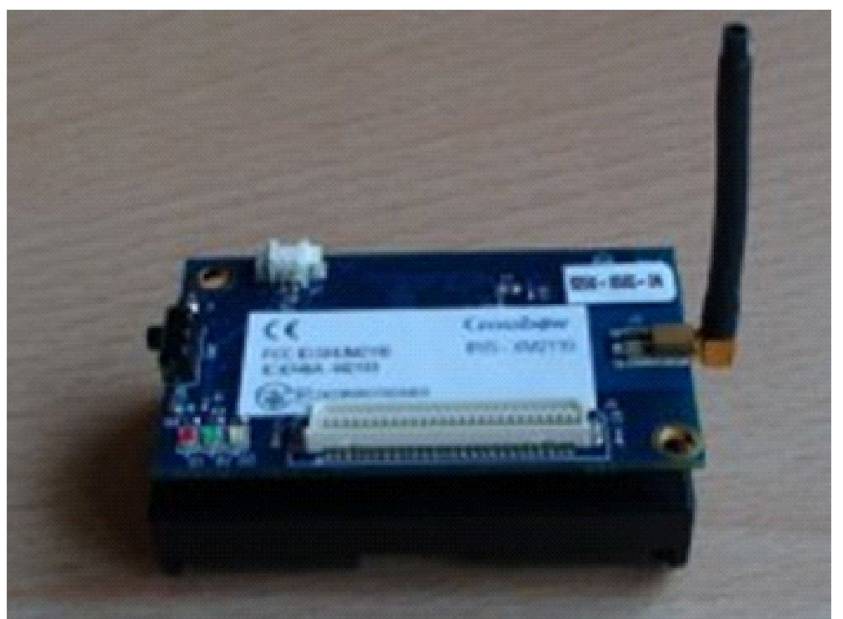

Figura 4. (A) Placa de aquisição MDA300CA; (B) nó sensor com plataforma IRIS ${ }^{\circledR}$ 
No software MoteView ${ }^{\circledR}$ foram fornecidos os dados de qualidade em parâmetros de qualidade da transmissão (Quality_Tx) e qualidade da recepção (Quality_Rx). Esses parâmetros são grandezas adimensionais expressos em valores de 0 a 15 que correspondem à qualidade de 0 a $100 \%$, respectivamente, faixa em que 0 corresponde a uma qualidade baixa e o número de transmissões necessárias para conseguir o estabelecimento da comunicação é elevado; 15 corresponde a uma qualidade alta e o número de transmissões necessárias para conseguir o estabelecimento da comunicação é igual a 1.

O termo "qualidade da transmissão" está relacionado com a passagem de sinal no sentido nó sensor-estação base; já a "qualidade da recepção" fornece o nível do enlace no sentido contrário, isto é, estação base-nó sensor.

O experimento foi dividido em três fases:

Fase 1: Alcance entre nós e estação base - Nesta fase observou-se o alcance do sinal entre o nó (localizado no interior da casa de vegetação) e a estação base fazendo variar o valor da potência de sinal (RF Power). Para isso, um nó sensor foi posicionado no centro da casa de vegetação numa altura de 1,5 m, enquanto a estação base era deslocada, sempre a altura de 1,5 m. Adistância máxima de alcance foi registrada e tomada como critério para definir os valores de potência de sinal que seriam utilizados nas fases posteriores ao experimento.

Fase 2: Medição do tempo de descarga da bateria em relação à potência de sinal - Nesta fase verificou-se o tempo de descarga da bateria para os valores de potência de sinal, determinados na Fase 1; para isto, as potências de sinal foram programadas no software MoteConfig® para -9,2 e -12,2 dBm, sendo então realizados dois testes. Através do software MoteView®, o nó foi configurado para fornecer dados de temperatura e umidade a cada $3 \mathrm{~s}$. Foram utilizadas duas pilhas do tipo AA com 1,5 V. A Estação Base foi deixada a $1 \mathrm{~m}$ de distância do nó sensor; desta forma, para a determinação do tempo de descarga o sistema programado foi deixado em pleno funcionamento até que o nó sensor parasse de funcionar.

Fase 3: Qualidade do sinal entre nó e estação base - Para comparação e análise dos dados utilizou-se a escala de qualidade de transmissão e recepção, obtendo-se a seguinte classificação:

- Acima de 13: muito alta

- Entre 12 e 9: alta

- Entre 8 e 5: média

- Abaixo de 4: baixa

Foram realizados testes para avaliar a influência do reposicionamento do nó sensor no interior da casa de vegetação na perda de qualidade de sinal. Nesta fase, foi realizada a programação do nó para mudar o valor de potência de sinal (-9,2 e -12,2 dBm). A posição da estação base foi fixada em uma sala com fonte de energia, localizado próximo à casa de vegetação. O nó sensor foi deslocado para 7 pontos (Figura 5), sendo que em cada posição foi fixado em 4 alturas diferentes, totalizando 27 pontos de medição. Na posição 4 (centro), não foi possível realizar a medição a $0 \mathrm{~m}$ de altura, já que a presença da bandeja com morangueiros significou um obstáculo que impediu o estabelecimento da comunicação. Os diferentes pontos de posicionamento dos nós permitiram verificar a influência do ambiente e da distância nos aspectos que envolvem a qualidade do sinal. Assim, os nós sensores foram posicionado da seguinte forma: base;

- Pontos 1 e 2: sem nenhuma bancada entre ele e a estação

- Pontos 3, 4 e 5: em locais próximos ao centro da casa de vegetação e com presença de bancada;

- Pontos 6 e 7: próximo ao sistema de resfriamento evaporativo (meio condutivo) com presença das bancadas entre nó e estação base.

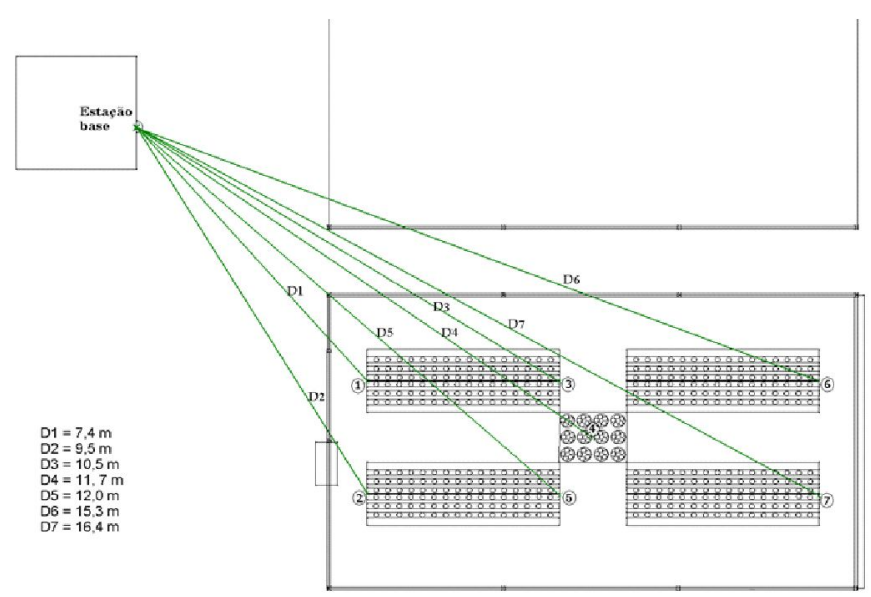

Figura 5. Pontos de medição e distâncias até a estação base

As alturas de cada ponto de coleta de dados $(0,0,0,8,1,3 \mathrm{e}$ $2,0 \mathrm{~m}$ ) foram escolhidas com base na estrutura da bancada e da casa de vegetação. Assim:

- 0,0 m: o sensor foi colocado no piso

- 0,8 m: sensor localizado próximo à altura da segunda linha de morangueiros

- 1,3 m: sensor colocado na mesma altura da estação base e no topo da bancada

- 2,0 m: o sensor foi posicionado próximo à tela de sombreamento

Em cada ponto o nó sensor permaneceu fornecendo pacotes de dados a cada $2 \mathrm{~min}$, durante $20 \mathrm{~min}$. Ou seja, em cada ponto eram gerados 10 pacotes que incluem os dados de qualidade do sinal (LQI). Com esses valores foram calculadas as médias e os desvios padrão de qualidade na transmissão e recepção do sinal (Tx, e Rx). Todo o procedimento da Fase 3 foi realizado duas vezes para as potências de sinal de $-12,2 \mathrm{e}$ $-9,2 \mathrm{dBm}$.

\section{RESULTADOS E DISCUSSÃO}

\section{Fase 1: Alcance nó-estação base}

Os resultados de alcance máximo entre o Nó Sensor e a Estação Base, obtidos para os valores de potência de sinal programados no software MoteConfig® são mostrados na Tabela 1.

Os demais valores de potência de sinal, disponíveis para programação no software MoteConfig®, por serem valores de potências maiores, obtinham um alcance máximo que 
Tabela 1. Valores de potência de sinal e de distância máxima entre estação base e nó sensor

\begin{tabular}{cc}
\hline RF Power (dBm) & Distância (m) \\
$-9,2$ & 30 \\
$-12,2$ & 15 \\
$-17,2$ & 5 \\
\hline
\end{tabular}

ultrapassava os limites da área do experimento, ou seja, foi maior ou igual a $35 \mathrm{~m}$ porém, como além deste limite o terreno era acidentado com presença de vegetação e de estruturas metálicas, não foi possível registrar os valores de alcance máximo de sinal para toda a escala de potência de sinal fornecida no MoteConfig ${ }^{\circledR}$. O teste mostrou que a distância máxima para a potência de sinal de $-17,2 \mathrm{dBm}$ era insuficiente para a realização dos testes.

Por sua vez, com o valor de $-12,2 \mathrm{dBm}$, verificou-se o alcance máximo próximo do limite dos testes realizados da Fase 3. Entretanto, para uma potência de sinal com valor de $-9,2 \mathrm{dBm}$ o alcance foi de quase $50 \%$ superior ao necessário. Por essa razão, com base nos resultados mostrados na Tabela 1 , foi determinado que os testes de qualidade fossem realizados para duas programações de potência de sinal: -9,2 e -12,2 dBm.

\section{Fase 2: Medição do tempo de descarga da bateria em relaçãoà potência de sinal}

O resultado obtido através dos testes é apresentado na Figura 6.

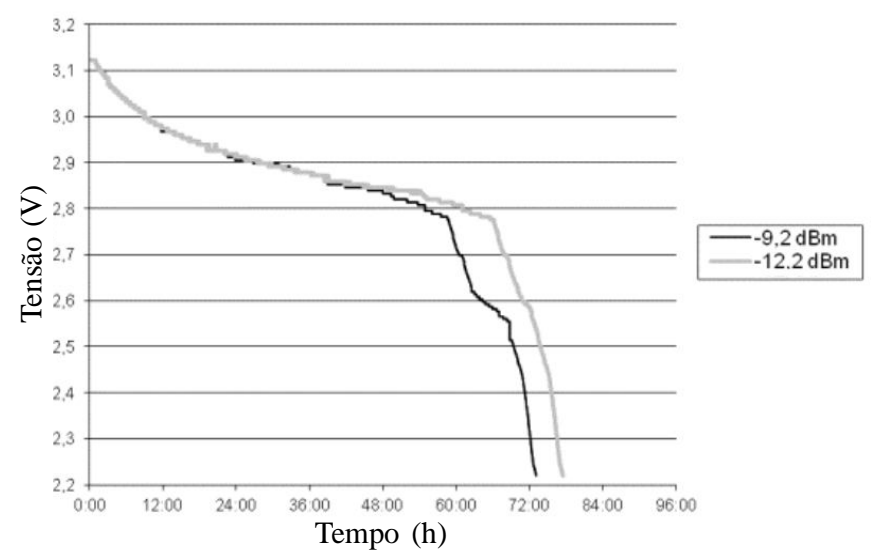

Figura 6. Tempo de descarga da bateria

A Figura 6 mostra uma diferença de aproximadamente $4 \mathrm{~h}$ entre a descarga da bateria para uma potência de sinal de $-9,2$ $\mathrm{dBm}$ e a descarga para uma potência de sinal de $-12,2 \mathrm{dBm}$ observando-se, daí, que a utilização de uma potência de sinal mais baixa, nessas condições, traria economia de energia para o sistema de aquisição.

\section{Fase 3: Qualidade do sinal entre nó e estação base}

A - Qualidade da transmissão

As médias dos dados de qualidade da transmissão da casa de vegetação para as potência de sinal de $-9,2 \mathrm{e}-12,2 \mathrm{dBm}$, são mostradas na Tabela 2A e 2B, respectivamente.

Da Tabela 2A observa-se que em duas posições, ambas a $0,8 \mathrm{~m}$ de altura, há queda na qualidade do sinal transmitido, que saiu da faixa de qualidade considerada "muito alta" para
Tabela 2. M édia dos dados de qualidade na transmissão do sinal para potências de sinal de -9,2 e - 12,2 dBm

\begin{tabular}{cccccccr}
\hline Altura & \multicolumn{7}{c}{ Distância $(\mathbf{m})$} \\
\cline { 2 - 8 }$(\mathbf{m})$ & $\mathbf{7 , 4}$ & $\mathbf{9 , 5}$ & $\mathbf{1 0 , 5}$ & $\mathbf{1 1 , 7}$ & $\mathbf{1 2 , 0}$ & $\mathbf{1 5 , 3}$ & $\mathbf{1 6 , 4}$ \\
0,0 & 15 & 13 & 15 & - & 15 & 15 & 15 \\
0,8 & 15 & 15 & 15 & 15 & 15 & 11 & 7 \\
1,3 & 15 & 15 & 15 & 15 & 15 & 15 & 15 \\
2,0 & 15 & 15 & 15 & 15 & 15 & 15 & 15 \\
\hline \multicolumn{7}{c}{ B. Potência de sinal de - $12,2 \mathrm{dBm}$} \\
0,0 & 10 & 15 & 13 & - & 13 & 15 & 13 \\
0,8 & 15 & 15 & 12 & 15 & 15 & 0 & 6 \\
1,3 & 15 & 15 & 15 & 10 & 15 & 15 & 13 \\
2,0 & 15 & 15 & 15 & 15 & 0 & 15 & 9 \\
\hline
\end{tabular}

qualidade "alta" (valor de 11) e qualidade "média" (valor de 7). Essas duas posições, além de estarem localizadas nas duas maiores distâncias em relação à estação base (15,3 e 16,4 m), estavam situadas numa altura próxima à segunda linha de morangueiros. Comparando com os resultados obtidos com os nós para as alturas de 0,0,1,3 e 2,0 m, há um volume maior de vegetação entre o sensor e a estação base na altura de $0,8 \mathrm{~m}$. Nota-se que, além da distância, o volume de plantas também contribuiu na diminuição da qualidade no sinal transmitido já que são obstáculos que introduzem atenuação no nível do sinal.

A influência do volume de vegetação na transmissão de sinal já foi testada por outros autores, para diferentes tipos de culturas. Zhang (2004), por exemplo, estudou o alcance da transmissão de um dispositivo com tecnologia Bluetooth 2,4 $\mathrm{GHz}$ e encontrou, para o solo nu, a soja e o milho, alturas ótimas de sensores de 1,4,1,7 e $4 \mathrm{~m}$, respectivamente. Goense $\&$ Thelen (2005) mostraram que para uma lavoura de batatas os sinais foram mais fracos quando se deu o desenvolvimento total da planta, caso em que a altura do sensor era de $0,295 \mathrm{~m}$. Tate et al. (2008) posicionaram transceptores com alturas de antena de 1,5 e 2,0 $\mathrm{m}$ em um campo de milho com 2,5 $\mathrm{m}$ de altura e distância de $100 \mathrm{~m}$. Este teste mostrou haver queda de potência média de sinal de $10 \mathrm{dBm}$ quando os transceptores foram colocados nas linhas de plantação. Hebel (2006) mostrou que a atenuação e o sinal variância da potência eram dependentes de linha de perdas de visão e alturas menores que o raio da zona de Fresnel. De acordo com estudos realizados por Giacomin \& Vasconcelos (2006) a presença de vegetação causa diminuição na intensidade do sinal recebido; assim, todos os trabalhos citados mostram que há influência significativa da vegetação na transmissão de dados de uma RSSF, o que justifica a diminuição da qualidade de sinal na altura de $0,8 \mathrm{~m}$.

A Tabela 2B mostra que os valores da média de qualidade na transmissão para uma potência de $-12,2 \mathrm{dBm}$ são classificados "baixo" em duas posições (a 12,0 e 15,3 m de distância) e razoável em uma posição (a 16,4 m de distância). Assim, novamente as duas posições mais distantes, em que o sensor foi colocado a $0,8 \mathrm{~m}$ de altura do piso, apresentaram valores de qualidade abaixo do registrado nos demais lugares.

Além do mais e comparando os valores obtidos nas Tabelas $2 \mathrm{~A}$ e $2 \mathrm{~B}$, observa-se que mais pontos saíram da faixa de qualidade "muito alta" e, em duas posições, a qualidade do sinal chegou a assumir valores nulos (distância de 12 e 15,3 m). 
O valor nulo de qualidade assumido na posição de $12,0 \mathrm{~m}$ de distância e $0,8 \mathrm{~m}$ de altura é justificado pela proximidade com a tela de sombreamento e a presença da porta e de uma coluna de metal entre o nó sensor e a estação base, sinalizando que a alteração da potência de sinal influencia diretamente na qualidade de sinal para alguns dos pontos da casa de vegetação. Os pontos mais afetados estão localizados sobretudo nas distâncias maiores de $12 \mathrm{~m}$.

Os desvios padrão da qualidade da transmissão de sinal recolhidos na casa de vegetação para potências de sinal de 9,2 e-12,2 dBm, são mostrados nas Tabelas 3A e 3B.

Tabela 3. Desvios padrão dos dados de qualidade na transmissão do sinal para potências de sinal de -9,2 e $-12,2 \mathrm{dBm}$

\begin{tabular}{cccccccc}
\hline Altura & \multicolumn{7}{c}{ Distância $(\mathbf{m})$} \\
\cline { 2 - 8 }$(\mathbf{m})$ & $\mathbf{7 , 4}$ & $\mathbf{9 , 5}$ & $\mathbf{1 0 , 5}$ & $\mathbf{1 1 , 7}$ & $\mathbf{1 2 , 0}$ & $\mathbf{1 5 , 3}$ & $\mathbf{1 6 , 4}$ \\
0,0 & A. Desvio padrão & para potência de & inal de & $-\mathbf{9 , 2}$ & $\mathrm{dBm}$ \\
0,8 & 0 & 4 & 0 & - & 0 & 0 & 1 \\
1,3 & 0 & 0 & 0 & 0 & 0 & 1 & 1 \\
2,0 & 0 & 1 & 1 & 0 & 1 & 0 & 0 \\
\hline \multicolumn{7}{c}{ B. Desvio padrão para potência de sinal de $-12,2 \mathrm{dBm}$} \\
0,0 & 7 & 0 & 5 & - & 6 & 0 & \\
0,8 & 0 & 0 & 5 & 0 & 1 & 0 & 6 \\
1,3 & 0 & 0 & 0 & 5 & 1 & 0 & 5 \\
2,0 & 0 & 0 & 0 & 0 & 0 & 0 & 5 \\
\hline
\end{tabular}

Na Tabela 3A é possível observar que somente quando o sensor estava posicionado no piso (0,0 $\mathrm{m}$ de altura) a uma distância de 9,5 m da estação base, o desvio padrão para valores de qualidade recolhidos no intervalo de 20 min assumiu um valor igual a 4; assim, em virtude da instabilidade da qualidade do sinal este pode ser considerado um ponto crítico da propagação de sinais no interior da casa de vegetação. Considerando a frequência de trabalho de $2,4 \mathrm{GHz}$, mencionada instabilidade do sinal nesta posição do nó, pode ser atribuída a desvanecimentos provocados pela presença de reflexões no interior da casa de vegetação. Tais reflexões produzem réplicas de sinais que se interferem, construtiva e negativamente, de forma aleatória na antena do nó sensor e afetam a qualidade tanto da transmissão como da recepção. A eventual presença de motores de indução operantes na frequência de $60 \mathrm{~Hz}$ não gera harmônicos que alcancem a faixa de $2,4 \mathrm{GHz}$ e, portanto, estes não são fontes de interferência.

Os valores apresentados na Tabela 3B mostram que, em 11 posições, no intervalo de tempo de 20 minutos os valores coletados de qualidade de sinal foram dispersos em relação à média, evento que ocorre com menor frequência para os desvios padrão obtidos com uma Potência de sinal de -9,2 dBm (Tabela 3A) e, portanto, é mais fácil manter o mesmo nível de qualidade de transmissão quando é utilizada a potência de sinal de -9,2 $\mathrm{dBm}$, em comparação com a potência de $-12,2 \mathrm{dBm}$.

\section{B - Qualidade da recepção}

As médias dos dados de qualidade da recepção de sinal da casa de vegetação, para Potências de Sinal de -9,2 e-12,2 dBm, são mostradas nas Tabelas 4A e 4B.
Tabela 4. Média dos dados de qualidade na recepção do sinal para potências de sinal de -9,2 e -12,2 dBm

\begin{tabular}{cccccccc}
\hline Altura & \multicolumn{7}{c}{ Distância (m) } \\
\cline { 2 - 8 } (m) & $\mathbf{7 , 4}$ & $\mathbf{9 , 5}$ & $\mathbf{1 0 , 5}$ & $\mathbf{1 1 , 7}$ & $\mathbf{1 2 , 0}$ & $\mathbf{1 5 , 3}$ & $\mathbf{1 6 , 4}$ \\
\multicolumn{7}{c}{ A. Potência de sinal de -9,2 dBm } \\
0,0 & 15 & 14 & 15 & - & 15 & 15 & 15 \\
0,8 & 15 & 15 & 15 & 15 & 15 & 14 & 15 \\
1,3 & 15 & 15 & 15 & 15 & 15 & 15 & 15 \\
2,0 & 15 & 15 & 15 & 15 & 15 & 15 & 15 \\
\hline \multicolumn{7}{c}{ B. Potência de sinal de - $12,2 \mathrm{dBm}$} \\
0,0 & 10 & 15 & 13 & - & 14 & 15 & 12 \\
0,8 & 15 & 15 & 13 & 15 & 14 & 2 & 10 \\
1,3 & 15 & 15 & 13 & 11 & 15 & 15 & 14 \\
2,0 & 15 & 12 & 15 & 15 & 5 & 15 & 11 \\
\hline
\end{tabular}

Os valores das médias da qualidade na recepção mostraramse excelentes em todos os pontos de amostragem, sem que nenhum valor fosse afetado pela distância nem pela vegetação.

Novamente, tal como se verifica nas Tabelas $2 \mathrm{~A}$ e B, as Tabelas 4A e 4B mostram que ocorre um aumento do número de pontos com qualidade abaixo da classificação "muito alto", quando o sinal é programado para uma potência de -12,2 dBm. Além disso, ocorreram diminuições significativas no valor da qualidade nos pontos localizados a 12 e 15,3 m.

Semelhante ao que ocorre na Tabela $2 \mathrm{~B}$, na posição de $12 \mathrm{~m}$ de distância e $2 \mathrm{~m}$ de altura apresenta um valor de qualidade de sinal inferior, classificado "médio", situação pode ser justificada pela proximidade com a tela de sombreamento e pela presença da porta e de uma coluna de metal entre este ponto e a estação base.

Os desvios padrão da qualidade da recepção de sinal recolhidos da casa de vegetação para uma Potência de sinal de $-9,2$ e -12,2 dBm são mostrados nas Tabelas 5A e 5B.

Tabela 5. Desvios padrão dos dados de qualidade na recepção do sinal para potências de sinal de -9,2 e-12,2 $\mathrm{dBm}$

\begin{tabular}{|c|c|c|c|c|c|c|c|}
\hline \multirow{2}{*}{$\begin{array}{l}\text { Altura } \\
\text { (m) }\end{array}$} & \multicolumn{7}{|c|}{ Distância (m) } \\
\hline & 7,4 & 9,5 & 10,5 & 11,7 & 12,0 & 15,3 & 16,4 \\
\hline \multicolumn{8}{|c|}{ A. Desvio padrão para Potência de sinal de $-9,2 \mathrm{dBm}$} \\
\hline 0,0 & 0 & 2 & 0 & - & 0 & 0 & 0 \\
\hline 0,8 & 0 & 0 & 0 & 0 & 0 & 1 & 0 \\
\hline 1,3 & 0 & 1 & 0 & 0 & 0 & 0 & 0 \\
\hline 2,0 & 0 & 1 & 0 & 0 & 0 & 0 & 0 \\
\hline \multicolumn{8}{|c|}{ B. Desvio padrão para Potência de sinal de $-12,2 \mathrm{dBm}$} \\
\hline 0,0 & 6 & 0 & 3 & & 3 & 0 & 0 \\
\hline 0,8 & 0 & 0 & 1 & 0 & 1 & 1 & 0 \\
\hline 1,3 & 0 & 0 & 0 & 4 & 1 & 1 & 0 \\
\hline 2,0 & 0 & 5 & 0 & 1 & 7 & 0 & 0 \\
\hline
\end{tabular}

Nas Tabelas 5A e 5B são apresentados valores de desvio maiores que zero em 4 e 12 posições, respectivamente.

Os valores apresentados na Tabela 5B mostram que em 12 posições no intervalo de tempo de $20 \mathrm{~min}$, os valores coletados de qualidade de sinal na recepção foram dispersos em relação à média cuja ocorrência é devida à menor frequência para os desvios padrão obtidos com uma potência de sinal de $-9,2 \mathrm{dBm}$ 
(Tabela 5A); daí se comprova que, outra vez, ocorreu uma variação maior dos valores de qualidade de recepção em torno da média quando utilizada uma Potência de sinal menor (-12,2 $\mathrm{dBm}$ ) dispersão esta devida aos efeitos combinados de uma potência menor de operação e à presença de desvanecimentos aleatórios nessas posições, conforme comentado na seção anterior; desta forma e com tal potência é mais difícil manter a qualidade de recepção e de transmissão estabilizada, como ocorre para a potência mais alta, de $-9,2 \mathrm{dBm}$.

\section{ConClusÕES}

1. A potência de sinal com valor de $-12,2 \mathrm{dBm}$ é suficiente para manter a transmissão entre estação base e nó sensor.

2. Para uma potência de sinal de $-9,2 \mathrm{dBm}$ constata-se que a rede possui qualidade classificada "muito alta" na maior parte dos pontos.

3. Existe uma economia de energia ao se utilizar a potência de sinal de $-12,2 \mathrm{dBm}$.

\section{LITERATURA CITADA}

Anastasi, G.; Conti, M.; Francesco, M.; Passarella, A. Energy conservation in wireless sensor networks: A survey. Ad Hoc Networks. v.7, p.537-568. 2009.

Aziz, A.; Hasan, M. H.; Ismail, M. J.; Mehat, M.; Haron, N. S. Remote monitoring in agricultural greenhouse using wireless sensor and short message service (SMS). International Journal of Engineering \& Technology. v.9, p.1-12. 2009.

Giacomin, J. C., Vasconcelos, F. H. Wireless sensor network as a measurement tool in precision agriculture. In: Imeko World Congress - Metrology for a Sustainable Development, 18, 2006, Rio de Janeiro. Proceedings... Rio de Janeiro: IMEKO, 2006. p.17-22.

Goense, D.; Thelen, J. Wireless sensor networks for precise phytophthora decision support. In: European Conference on Precision Agriculture, 2005, Wageningen. Proceedings... Wageningen: Uppsala, v.5, p.1-9. 2005.

Hebel, M. A. Meeting wide-area agricultural data acquisition and control challenges through zigbee wireless network technology. In: World Congress Conference on Computers in Agriculture and Natural Resources, 2006, Orlando. Proceedings... Orlando: American Society of Agricultural and Biological Engineers, v.4, 2006. p.234-239.
Jackson, T.; Mansfield, K.; Saafi, M.; Colman, T.; Romine, P. Measuring soil temperature and moisture using wireless mems sensors. Journal Measurement. v.41, p.381-390. 2007.

Matijevics, I; Simon, J. Control of the greenhouse's microclimatic condition using wireless sensor network, Ipsi Journal, v.6, p.35-38. 2010.

Morais, R.; Fernandes, M. A.; Matos, S. G.; Serôdio, C., Ferreira, P.J. S. G., Reis, M. J. C. S. AZigbee multi-powered wireless acquisition device for remote sensing applications in precision viticulture. Computers and Electronics in Agriculture, v. 62, p.94-106. 2008.

Morais, R.; Valente, A.; Couto, C.; Correia, J. H. A Wireless RF CMOS mixed-signal interface for soil moisture measurements. Sensors and Actuators A: Physical. v.115, p.376-384. 2004.

Omid, M.; Shafaei, A. Temperature and relative humidity changes inside greenhouse. International Agrophysics, v.19, p.153-158. 2004.

Queiroz, T. M.; Botrel, T. A.; Frizzone, J. A. Desenvolvimento de software e hardware para irrigação de precisão usando pivô central. Engenharia Agrícola, v.28, p.44-54. 2008.

Ruiz-Altisent, M; Ruiz-Garcia, L; Moreda, G; Lu, R; HernandezSanchez, N; Correa, E; Diezma, B; Nicolai, B; Garcia-Ramos, J. Sensors for product characterization and quality of specialty crops - A review. Computers and Electronics in Agriculture. v.74, p.176-194, 2010.

Santos, I ; Souza, G.; Margalho, M., The use of underwater wireless networks in pisciculture at Amazon. International Journal of Computer Science Issues, v.7, p.112-116. 2010.

Serôdio, C.; Cunha, J. B.; Morais, R.; Couto, C. A.; Monteiro, J. L. A networked platform for agricultural management systems. Computers and Electronics in Agriculture, v.31, p.75-90. 2001.

Tate, R. F.; Hebel, M. A.; Watson, D.G. WSN Link budget analysis for precision agriculture. In: Annual International Meeting, Providence, 2008, Anais.... Providence : American Society of Agricultural and Biological Engineers, p.67866795, 2008.

Wang, N; Zhang, N; Wang, M. Wireless sensors in agriculture and food industry -Recent development and future perspective. Computers and Electronics in Agriculture, v.50, p.1-14. 2006.

Zhang, Z. Investigation of wireless sensor networks for precision agriculture. In: Annual International Meeting, 2004, Ottawa. Proceedings... Ottawa: American Society of Agricultural and Biological Engineers, 2004. p.31-35. 